International Journal of Network Security \& Its Applications (IJNSA), Vol.3, No.4, July 2011

\title{
WAVELET ThreSHOLDING APPROACH FOR IMAGE DENOISING
}

\author{
Pankaj Hedaoo ${ }^{1}$ and Swati S Godbole ${ }^{2}$ \\ ${ }^{1}$ Department of Electronics \& Telecommunication Engineering, \\ G. H. Raisoni College of Engineering, Nagpur, India. \\ pankaj.s.hedaoo@gmail.com \\ ${ }^{2}$ Department of Electronics \& Telecommunication Engineering, \\ G. H. Raisoni College of Engineering, Nagpur, India \\ godbole123eyahoo.com
}

\begin{abstract}
The original image corrupted by Gaussian noise is a long established problem in signal or image processing .This noise is removed by using wavelet thresholding by focused on statistical modelling of wavelet coefficients and the optimal choice of thresholds called as image denoising. For the first part, threshold is driven in a Bayesian technique to use probabilistic model of the image wavelet coefficients that are dependent on the higher order moments of generalized Gaussian distribution (GGD) in image processing applications. The proposed threshold is very simple. Experimental results show that the proposed method is called BayesShrink, is typically within 5\% of the MSE of the best soft-thresholding benchmark with the image. It outperforms Donoho and Johnston Sure Shrink.
\end{abstract}

The second part of the paper is attempt to claim on lossy compression can be used for image denoising .thus achieving the image compression \& image denoising simultaneously. The parameter is choosing based on a criterion derived from Rissanen's minimum description length (MDL) principle. Experiments show that this compression \& denoise method does indeed remove noise significantly, especially for large noise power.

\section{KEYWORDS}

Image denoising, Wavelet Thresholding, Noise categories, Proposed Method.

\section{INTRODUCTION}

Denoising of images corrupted by additive white Gaussian noise (AWGN) are classical problem in image processing. The distortions of images by noise are common during its acquisition, processing, compression, transmission, and reproduction. In the past two decades several successful articles focused on removing the noise from the image to increase the overall quality of the processed image. Especially the case of additive white Gaussian noise a number of techniques using wavelet-based thresholding . Donoho and Johnston proposed hard and soft thresholding methods for Denoising. This scheme exterminates many wavelet coefficients that might contain useful image information. However, the major problem with both methods is the choice of a suitable threshold value. The definition of coefficient independent threshold given by Donoho and Johnston depends on the noise power and the size of the image. In practice, however one deals with images of finite size where the applicability of such a theoretical result is rather questionable. In addition, most signals show spatially non-uniform energy distribution, which motivate the choice of a non-uniform threshold. Linear filtering is an efficient technique to deal with additive noise while non-linear filters are efficient to deal with the multiplicative and function based noise.

Wavelet shrinkage method proposed by Donoho [1]-[3] is the pioneering work for signal denoising using the wavelet transform. The method described in [3] provides a mini-max 
International Journal of Network Security \& Its Applications (IJNSA), Vol.3, No.4, July 2011

optimal solution. Another criterion commonly used is the mean squared error (MSE), even though it does not match well with the characteristics of the human visual system [4]. The discrete wavelet transform (DWT) used in image denoising can be real/complex-valued.

The intuition behind using lossless compression for denoising may be explained as follows. A signal typically has structural correlations that a good coder can exploit to yield to the point of representation. White noise, however, doesn't have structural redundancy and thus it is not easily compressible. Hence, a good compression method can provide a suitable model for distinguishing between signal and noise. Lossy compression and denoising can easily be seen when one examines the similarity between thresholding and quantization, the latter of which is a necessary step in a practical lossy coder. The quantization of wavelet coefficients with a zero-zone is an approximation to the thresholding function (see Fig. 1). Thus, provided that the quantization outside of the zero-zone does not introduce significant distortion, it follows that wavelet-based lossless compression achieves denoising. With this connection in mind, this paper is about wavelet thresholding for image denoising and also for lossless compression. The threshold choice aids the lossy coder to choose its zero-zone, and the resulting coder achieves simultaneous denoising and compression if such property is required.

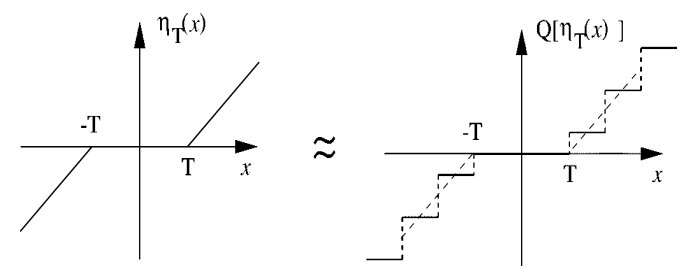

Figure. 1. The thresholding function approximation by quantization with a zero-zone

Many wavelet based thresholding techniques like Visu shrink, Oracle Shrink, Normal shrink have proved better efficiency in image denoising. For image denoising, however, Visu Shrink is known to yield overly smoothed images. This is because its threshold choice, $\sqrt{2} \log M$ (called the universat threshold and is the noise variance), can be unwarrantedly large due to its dependence on the number of samples, which is more than 105 for a typical test image of size 256*256 . Sure Shrink uses a hybrid of the universal threshold and the Sure threshold, derived from minimizing Stein's unbiased risk estimator has been shown to perform well (6). In this paper, we propose a framework and a near-optimal threshold more suitable for image denoising based on Bayesian analyzing the statistical parameters of the wavelet coefficients that outperforms the traditional ones, improving the denoised results significantly

\section{WAVELET THRESHOLDING}

Wavelet Thresholding is very simple non-linear technique, which operates on one wavelet coefficient at a time. In its most basic form, each coefficient is threshold by compare against threshold, if the coefficient is smaller than threshold, set to zero; otherwise it is kept or modified. Replacing the all small noisy coefficients by zero and inverse wavelet transform on the result may lead to reconstruction with the essential signal characteristics and with less noise. Wavelet thresholding involves threes steps A linear discrete wavelet transform, nonlinear thresholding Step \& a linear inverse wavelet transform.

Let us consider a signal $\left\{x_{i j}, i, j=1,2 \ldots \mathrm{N}\right\}$ denote the $\mathrm{N} \mathrm{X} \mathrm{N}$ matrix of the original image to be recovered and $\mathrm{N}$ is some integer power of 2. During transmission the signal is corrupted by independent and identically distributed (i.i.d) zero mean, white Gaussian Noise $z_{i j}$ with standard deviation $\sigma$ i.e. $z_{i j} \sim M(0, \sigma 2)$ as follows.

$$
y_{i j}=x_{i j}+z_{i j}
$$


From this noisy signal $y$, we want to find an approximation $x_{i j}$. The goal is to estimate the signal $x_{i j}$ from noisy observations $y_{i j}$ such that Mean Squared error (MSE) is minimum. I.e.

$$
\|\mathrm{X}-\overline{\mathrm{X}}\|^{2}=1 / \mathrm{M} \sum_{i=0}^{N-1}\left(\mathrm{X}_{\mathrm{i}}-\overline{\mathrm{X}}_{\mathrm{i}}\right)^{2}
$$

Let $\mathrm{W}$ and $\mathrm{W}^{-1}$ denote the two-dimensional orthogonal discrete wavelet transform (DWT) matrix and its IDWT respectively. Then equation (1) can be written as

$$
d_{i j}=c_{i j}+\varepsilon_{\mathrm{ij}}
$$

With $d=W y, c=W x, \varepsilon=W z$.Since $W$ is orthogonal transform, $\varepsilon j$ is also an i.i.d Gaussian random variable with $i j \varepsilon \approx(0, \sigma 2)$.Now $\mathrm{T}$ (.) be the wavelet thresholding function then the wavelet thresholding based Denoising scheme can be expressed as $X=W^{-1}(T(W y))$ wavelet transform of noisy signal should be taken first and then thresholding function is applied on it. Finally the output should be undergone inverse wavelet transformation to obtain the estimate $x$. There are two thresholds frequently used, i.e. hard threshold, soft threshold. The hardthresholding function is described as

$$
\begin{array}{rlrl}
f_{h}(x) & =x & \text { if } x \geq \lambda \\
& =0 & & \text { otherwise }
\end{array}
$$

The hard-thresholding function chooses all wavelet coefficients that are greater than the given threshold $\lambda$ and sets the others to zero. The threshold $\lambda$ is chosen according to the signal energy and the noise variance $\left(\sigma^{2}\right)$.

The soft-thresholding function has a somewhat different rule from the hard-thresholding function. It shrinks the wavelet coefficients by $\lambda$ towards zero,

$$
\begin{aligned}
f(x) & =\mathrm{x}-\lambda & & \text { if } x \geq \lambda \\
& =0 & & \text { if } x<\lambda \\
& =\mathrm{x}+\lambda & & \text { if } x \leq-\lambda
\end{aligned}
$$

The soft-thresholding rule is chosen over hard-thresholding, for the soft-thresholding method yields more visually pleasant images over hard thresholding.

\section{NOISE CATEGORIES}

The classification of noise relies mainly on the characterizing probabilistic specifications. There are the four types of noise categories in image processing

1. Gaussian noise

2. Salt and Pepper Noise

3. Poison noise

4. Speckle Noise

Any one of above noise uses for addition of noise.

\section{PROPOSED ALGORITHM}

4.1. Adaptive thresholding function:-This section focuses on the estimation of the GGD parameters, which in turn yields a data-driven estimate of that is adaptive to different sub band characteristics. The noise variance need to be estimate first. In some situations, it may be possible to measure based on information other than the corrupted image. If such is not the case, it is estimated from the sub band by the healthy median estimator.

Where

$$
\sigma^{\wedge}=\operatorname{Median}(|Y i j|) / .6745--(1)
$$

$$
\mathrm{Y}_{\mathrm{ij}} € \text { subbandHH } \mathrm{H}_{1}
$$


The parameter does not explicitly enter into the expression $\mathrm{T}_{\mathrm{B}}\left(\sigma_{\mathrm{x}}\right)$ of, only the signal standard deviation $\left(\sigma_{\mathrm{x}}\right) . \mathrm{T}_{\mathrm{B}}$ is the optimal threshold function. Therefore it suffices to estimate directly $\sigma_{\mathrm{x}}$ or $\sigma \times 2$. Recall the observation model is $\mathrm{Y}=\mathrm{X}+\mathrm{U}$, with $\mathrm{X}$ and $\mathrm{U}$ independent of each other, Where $\mathrm{n} \mathrm{x} \mathrm{n}$ is the size of the sub band under consideration. Thus

$$
\begin{gathered}
\mathrm{T}_{\mathrm{B}}^{\wedge}\left(\sigma_{\mathrm{x}}\right)=\frac{\sigma^{\wedge} 2}{\sigma^{\wedge} \mathrm{x}} \\
\text { Where } \\
\sigma_{\mathrm{y}}^{2}=\sigma^{2}+\sigma^{2} \\
\sigma_{\mathrm{x}}^{\wedge}=\sqrt{\max \left(\sigma^{\wedge}{ }_{\mathrm{y}}-\sigma^{\wedge}, 0\right)}
\end{gathered}
$$

Where $\sigma_{\mathrm{y}}^{2}$ is the variance of $\mathrm{Y}$. Since $\mathrm{Y}$ is modelled as zero-mean, $\sigma_{\mathrm{y}}^{2}$ can be found empirically by

$$
\begin{gathered}
\sigma_{\mathrm{y}}^{\wedge 2}=\frac{1}{n} 2 \sum_{i, j=1}^{n}\left(\mathrm{X}_{\mathrm{ij}}{ }^{2}\right)----(5) \\
\mathrm{T}_{\mathrm{B}}^{\wedge}\left(\sigma_{\mathrm{x}}\right)=\max \left(\left|\mathrm{y}_{\mathrm{ij}}\right|\right)------(6)
\end{gathered}
$$

The above equation shows the probalistics function which is proposed by scientist bayes .so, this method is called as B Wavelet Thresholding Bayesian shrink. And below equation shows the optimal threshold.

$$
\mathrm{T}_{\mathrm{B}}^{\wedge}\left(\sigma_{\mathrm{x}}\right)=\frac{\sigma^{\wedge} 2}{\sigma^{\wedge} \mathrm{x}}
$$

\subsection{Flowchart \& Experimental Results}

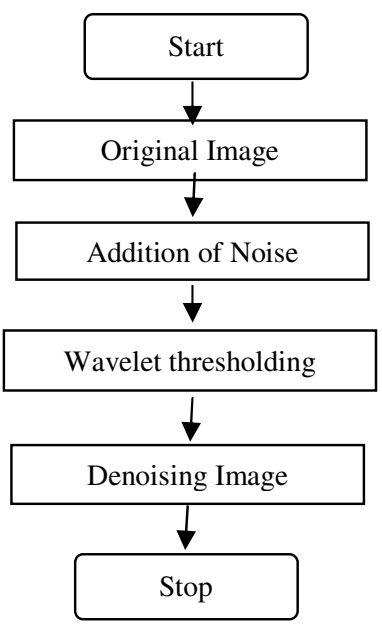




\subsection{Results}

\begin{tabular}{|c|c|c|c|c|}
\hline \multirow{2}{*}{ Image } & \multicolumn{4}{|c|}{ Peak Signal To Noise Ratio in } \\
db(PSNR)
\end{tabular}

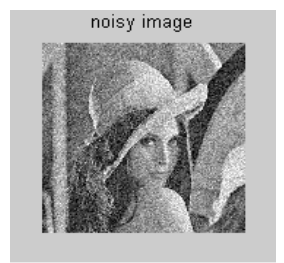

Noisy Lena at $\sigma=30$

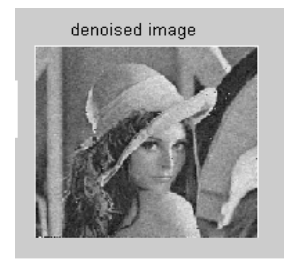

soft thresholding

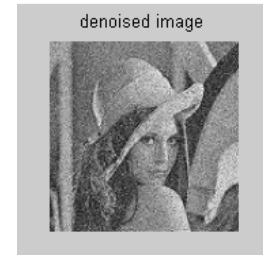

Hard thresholding

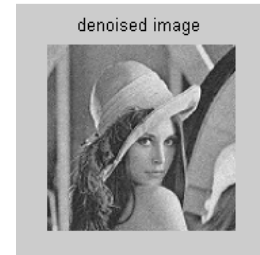

proposed Shrink method

Figure. 2. Comparing the performance of (a) Noisy Lena at $\sigma=30$ with (b) soft thresholding (c) Hard thresholding (d) proposed Shrink method

\section{CONCLuSiON}

The main issues regarding image denoising were addressed in this paper. an adaptive threshold for wavelet thresholding images was proposed, based on the generalized Guassian distribution modelling of subband coefficients, and test results showed excellent performance . The results show that Proposed Shrink removes noise significantly. In this paper, we compare the results with soft thresholding, hard thresholding \& proposed method

\section{REFERENCES}

[1] D. L. Donoho and I. M. Johnstone, "Ideal spatial adaptation by wavelet shrinkage," Biometrika, vol. 81, no. 3, pp. 425-455, 1994.

[2] D. L. Donoho, "Denoising by soft-thresholding," IEEE Trans. Inf. Theory, vol. 41, no. 3, pp. 613-627, Mar. 1995.

[3] D. L. Donoho and I. M. Johnstone, "Adapting to unknown smoothness via wavelet shrinkage,” J. Amer. Statist. Assoc., vol. 90, no. 432, pp. 1200-1224, 1995

[4] D.L. Donoho, De-Noising by Soft Thresholding, IEEE Trans. Info. Theory 43, pp. 933-936, 1993.

[5] S. Grace Chang, Bin Yu and M. Vattereli, Wavelet Thresholding for Multiple Noisy Image Copies, IEEE Trans. Image Processing, vol. 9, pp.1631- 1635, Sept.2000

[6] Savita Gupta and Lakhwinder kaur, Wavelet Based Image Compression using Daubechies Filters, In proc. 8th National conference on communications, I.I.T. Bombay, NCC-2002.

[7] S. Grace Chang, Bin Yu and M. Vattereli, Adaptive Wavelet Thresholding for Image Denoising and Compression, IEEE Trans. Image Processing, vol. 9, pp. 1532-1546, Sept. 2000.

[8] D. L. Donoho and I. M. Johnstone,'Threshold selection for wavelet shrinkage of Noisy Data," Proc16th Annual International conference of IEEE Engg in Medicine and biology Society .Vol1, Page A24-A25 Baltimore, Maryland, 1994. 
International Journal of Network Security \& Its Applications (IJNSA), Vol.3, No.4, July 2011

[9] Osama K Al-shaykh and Russell M Mersereau,” Lossy Compressed Noisy image ," IEEE Trans. on signal processing 1998,vol7,No12 1641-1652

[10] B. K .Natrajan," filtering randam noise from deterministic signals via data Compression," IEEE Tranc. on signal processing,Vol.43,No11,pp2595-2605

[11] F. Ruggeri and B. Vidakovic,” A Bayesian decision theoretic approach to wavelet thresholding ,"preprint , Duke University ,Durham ,NC

[12] Y Chen and C Han ," adaptive wavelet threshold for Image denoising ,'IEEE Electron, Lett, Vol-11, no 10 May 2005

[13] Gao H. Y and Bruce A G," Wave shrink with firm shrinkage ," Technical report 39 statics Division of Math soft ,Inc 1996

[14] Gao H. Y ," Wavelet shrinkage denoising Using non-negative garrote ,'J compute Graph statist ,pp 469-488,1998

[15] S Chang, Bin Yu and M Vattereli," Spatially Adaptive Wavelet Thresholding with context Modelling for Image Denoising “, IEEE trans. Image processing Vol.9 pp1522-1530 sept 2000.

[16] S. Chang , Bin Yu and M Vettereli ," Wavelet Thresolding for multiple noisy Image copies," IEEE Trans Image Processing VOL 9.pp 1631-1635 Sept 2000

[17] D L Donoho and I. M. Johnstone," Adapting to unknown smoothness via Wavelet Shrinkage ,'Journal of American statical Assoc ,Vol 9.pp1631-1635 Sept 2000

\section{Authors}

Pankaj S Hedaoo was born in Nagpur, Maharashtra, on Dec. $27^{\text {th }}$, 1987 . He received B.E from the R. T. M. Nagpur University, in 2009 and he is pursuing M.Tech. from R. T. M. Nagpur University. Currently he is working as a Lecturer in G H Raisoni College of Engg. At Nagpur. His research interest includes Adaptive Image Processing, Digital Image Processing.

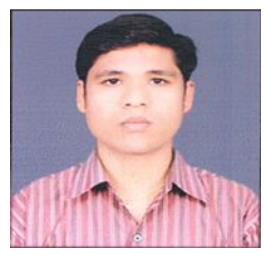

Swati S. Godbole was born in Nagpur, Maharashtra, on Sept. 28, 1978. She received BE M.Tech Degrees in Electronics Engineering from the R. T. M. Nagpur University, in 2000, 2006 and pursuing PhD from R. T. M. Nagpur University. She is currently working in G. H. Raisoni College of Engineering, Nagpur as a Assistant Professor. Her research interest includes Adaptive Signal Processing, Communication \& VHDL.

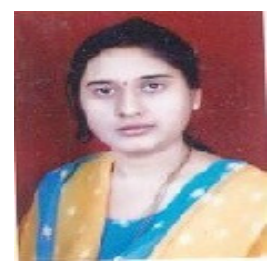

